\title{
SUMS OF ONE PRIME POWER AND TWO SQUARES OF PRIMES IN SHORT INTERVALS
}

\author{
ALESSANDRO LANGUASCO and ALESSANDRO ZACCAGNINI
}

\begin{abstract}
Aвstract. Let $k \geq 1$ be an integer. We prove that a suitable asymptotic formula for the average number of representations of integers $n=p_{1}^{k}+p_{2}^{2}+p_{3}^{2}$, where $p_{1}, p_{2}, p_{3}$ are prime numbers, holds in intervals shorter than the ones previously known.
\end{abstract}

\section{INTRODUCTION}

The problem of representing an integer as a sum of a prime power and of two prime squares is classical. It is conjectured that every sufficiently large $n$ subject to some congruence conditions can be represented as $n=p_{1}^{k}+p_{2}^{2}+p_{3}^{2}$, where $k \geq 1$ is an integer. Let now $N$ be a large integer and denote by $E_{k}(N)$ the cardinality of the set of integers not exceeding $N$ that satisfy the necessary congruence conditions but can not be represented as the sum of a $k$-th prime power and two prime squares. Several results about $E_{k}(N)$ were obtained; the first one who proved a non-trivial estimate for $E_{k}(N)$ was Hua [3]. Later Schwarz [13] and several other authors further improved such an estimate; we recall the contribution of Leung-Liu [8], Harman-Kumchev [2], Lü [10] and Li [9]. Let $\varepsilon>0$; so far the best known estimates are $E_{1}(N) \ll N^{1 / 3+\varepsilon}$ by Zhao [15], $E_{2}(N) \ll N^{17 / 20+\varepsilon}$ by Harman-Kumchev [2], $E_{3}(N) \ll N^{15 / 16+\varepsilon}$ and, for $k \geq 4$, $E_{k}(N) \ll N^{1-1 /\left(4 k^{2}\right)+\varepsilon}$ both by Brüdern [1]. Let

$$
r_{k}(n)=\sum_{p_{1}^{k}+p_{2}^{2}+p_{3}^{2}=n} \log p_{1} \log p_{2} \log p_{3} .
$$

In this paper we study the average behaviour of $r_{k}(n)$ over short intervals $[N, N+H], H=o(N)$ thus generalising our result in [5] which just deals with the case $k=1$.

Theorem 1. Let $N \geq 2,1 \leq H \leq N, k \geq 1$ be integers. Then, for every $\varepsilon>0$, there exists $C=C(\varepsilon)>0$ such that

$$
\sum_{n=N+1}^{N+H} r_{k}(n)=\frac{\pi}{4} H N^{1 / k}+\sigma_{k}\left(H N^{1 / k} \exp \left(-C\left(\frac{\log N}{\log \log N}\right)^{1 / 3}\right)\right) \text { as } N \rightarrow \infty,
$$

uniformly for $N^{1-5 /(6 k)+\varepsilon} \leq H \leq N^{1-\varepsilon}$ for $k \geq 2$ and $N^{7 / 12+\varepsilon} \leq H \leq N^{1-\varepsilon}$ for $k=1$.

It is worth remarking that the formula in Theorem 1 implies that every interval $[N, N+H]$ contains an integer which is a sum of a prime $k$-th power and two prime squares, where $N^{1-5 /(6 k)+\varepsilon} \leq H \leq N^{1-\varepsilon}$ for $k \geq 2$ and $N^{7 / 12+\varepsilon} \leq H \leq N^{1-\varepsilon}$ for $k=1$. In fact, for $k=1$ Zhao's estimate previously mentioned leads to better consequences than our Theorem 1 , but for $k \geq 2$ our result gives non-trivial information.

Assuming that the Riemann Hypothesis $(\mathrm{RH})$ holds, we prove that a suitable asymptotic formula for such an average of $r_{k}(n)$ holds in much shorter intervals. We need the following

2010 Mathematics Subject Classification. Primary 11 $\mathrm{P}_{32}$; Secondary $11 \mathrm{P}_{55},{ }_{11} \mathrm{P}_{5}$.

Key words and phrases. Waring-Goldbach problem, Laplace transforms. 
auxiliary function: let

$$
E(k):= \begin{cases}N^{3 / 2} \log N+H N^{3 / 4}(\log N)^{3 / 2} & \text { if } k=1 \\ N \log N+H N^{1 / 4}(\log N)^{2} & \text { if } k=2 \\ N^{5 / 6} \log N+H N^{1 / 4} \log N+H^{1 / 2} N^{1 / 2} \log N & \text { if } k=3 \\ N^{3 / 4+1 / k} \log N & \text { if } k \geq 4 .\end{cases}
$$

We have the following

Theorem 2. Assume the Riemann Hypothesis (RH). Let $N \geq 2,1 \leq H \leq N, k \geq 2$ be integers. We have

$$
\sum_{n=N+1}^{N+H} r_{k}(n)=\frac{\pi}{4} H N^{1 / k}+\sigma_{k}\left(H^{2} N^{1 / k-1}+H^{1 / 2} N^{1 / 2+1 /(2 k)}(\log N)^{2}+E(k)\right)
$$

as $N \rightarrow \infty$, uniformly for $\infty\left(N^{1-1 / k}(\log N)^{4}\right) \leq H \leq o(N)$, where $f=\infty(g)$ means $g=o(f)$, $\Gamma$ is Euler's function and $E(k)$ is defined in (2).

We remark that a version for $k=1$ of Theorem 2 was obtained in [5]. We further remark that the formula in Theorem 2 implies that every interval $[N, N+H]$ contains an integer which is a sum of a prime power and two prime squares, where $\infty\left(N^{1-1 / k}(\log N)^{4}\right) \leq H=o(N)$.

The proofs of both Theorems 1-2 use the original Hardy-Littlewood settings of the circle method to exploit the easier main term treatment they allow (comparing with the one which would follow using Lemmas 2.3 and 2.9 of Vaughan [14]).

It is worth remarking that the expected best result using circle method techniques is $H \geq$ $N^{1-1 / k}$; so our Theorem 2 , under the assumption of the Riemann Hypothesis, comes very close to this bound. We also obtained similar results in [6] and [7].

\section{Notation AND Lemmas}

$$
\begin{aligned}
& \text { Let } e(\alpha)=e^{2 \pi i \alpha}, \alpha \in[-1 / 2,1 / 2], L=\log N, z=1 / N-2 \pi i \alpha, \\
& \qquad \widetilde{S}_{\ell}(\alpha)=\sum_{n=1}^{\infty} \Lambda(n) e^{-n^{\ell} / N} e\left(n^{\ell} \alpha\right) \quad \text { and } \quad \widetilde{V}_{\ell}(\alpha)=\sum_{p=2}^{\infty} \log p e^{-p^{\ell} / N} e\left(p^{\ell} \alpha\right) .
\end{aligned}
$$

We remark that

$$
|z|^{-1} \ll \min \left(N,|\alpha|^{-1}\right)
$$

We further set

$$
U(\alpha, H)=\sum_{m=1}^{H} e(m \alpha)
$$

and, moreover, we also have the usual numerically explicit inequality

$$
|U(\alpha, H)| \leq \min \left(H ;|\alpha|^{-1}\right),
$$

see, e.g., on page 39 of Montgomery [11]. We list now the needed preliminary results.

Lemma 1 (Lemma 3 of [4]). Let $\ell \geq 1$ be an integer. Then $\left|\widetilde{S}_{\ell}(\alpha)-\widetilde{V}_{\ell}(\alpha)\right| \ll_{\ell} N^{1 /(2 \ell)}$.

Lemma 2. Let $\ell \geq 1$ be an integer, $N \geq 2$ and $\alpha \in[-1 / 2,1 / 2]$. Then

$$
\widetilde{S}_{\ell}(\alpha)=\frac{\Gamma(1 / \ell)}{\ell z^{1 / \ell}}-\frac{1}{\ell} \sum_{\rho} z^{-\rho / \ell} \Gamma(\rho / \ell)+\sigma_{\ell}(1),
$$


where $\rho=\beta+i \gamma$ runs over the non-trivial zeros of $\zeta(s)$.

Proof. It follows the line of Lemma 2 of [5]; we just correct an oversight in its proof. In eq. (5) on page 48 of [5] the term $-\sum_{m=1}^{\ell \sqrt{3} / 4} \Gamma(-2 m / \ell) z^{2 m / \ell}$ is missing. Its estimate is trivially $\ll_{\ell}|z|^{\sqrt{3} / 2} \ll_{\ell} 1$. Hence such an oversight does not affect the final result of Lemma 2 of [5].

Lemma 3 (Lemma 4 of [5]). Let $N$ be a positive integer and $\mu>0$. Then

$$
\int_{-1 / 2}^{1 / 2} z^{-\mu} e(-n \alpha) \mathrm{d} \alpha=e^{-n / N} \frac{n^{\mu-1}}{\Gamma(\mu)}+\sigma_{\mu}\left(\frac{1}{n}\right)
$$

uniformly for $n \geq 1$.

Lemma 4. Let $\varepsilon$ be an arbitrarily small positive constant, $\ell \geq 1$ be an integer, $N$ be a sufficiently large integer and $L=\log N$. Then there exists a positive constant $c_{1}=c_{1}(\varepsilon)$, which does not depend on $\ell$, such that

$$
\int_{-\xi}^{\xi}\left|\widetilde{S}_{\ell}(\alpha)-\frac{\Gamma(1 / \ell)}{\ell z^{1 / \ell}}\right|^{2} \mathrm{~d} \alpha \ll_{\ell} N^{2 / \ell-1} \exp \left(-c_{1}\left(\frac{L}{\log L}\right)^{1 / 3}\right)
$$

uniformly for $0 \leq \xi<N^{-1+5 /(6 \ell)-\varepsilon}$. Assuming $R H$ we get

$$
\int_{-\xi}^{\xi}\left|\widetilde{S}_{\ell}(\alpha)-\frac{\Gamma(1 / \ell)}{\ell z^{1 / \ell}}\right|^{2} \mathrm{~d} \alpha \ll_{\ell} N^{1 / \ell} \xi L^{2}
$$

uniformly for $0 \leq \xi \leq 1 / 2$.

Proof. It follows the line of Lemma 3 of [5] and Lemma 1 of [4]; we just correct an oversight in their proofs. Both eq. (8) on page 49 of [5] and eq. (6) on page 423 of [4] should read as

$$
\int_{1 / N}^{\xi}\left|\sum_{\rho: \gamma>0} z^{-\rho / \ell} \Gamma(\rho / \ell)\right|^{2} \mathrm{~d} \alpha \leq \sum_{k=1}^{K} \int_{\eta}^{2 \eta}\left|\sum_{\rho: \gamma>0} z^{-\rho / \ell} \Gamma(\rho / \ell)\right|^{2} \mathrm{~d} \alpha
$$

where $\eta=\eta_{k}=\xi / 2^{k}, 1 / N \leq \eta \leq \xi / 2$ and $K$ is a suitable integer satisfying $K=\sigma(L)$. The remaining part of the proofs are left untouched. Hence such oversights do not affect the final result of Lemma 3 of [5] and Lemma 1 of [4].

Lemma 5 (Lemma 2 of [4]). Let $\ell \geq 2$ be an integer and $0<\xi \leq 1 / 2$. Then

$$
\int_{-\xi}^{\xi}\left|\widetilde{S}_{\ell}(\alpha)\right|^{2} \mathrm{~d} \alpha \ll_{\ell} \xi N^{1 / \ell} L+\left\{\begin{array}{ll}
L^{2} & \text { if } \ell=2 \\
1 & \text { if } \ell>2
\end{array} \text { and } \int_{-\xi}^{\xi}\left|\widetilde{V}_{\ell}(\alpha)\right|^{2} \mathrm{~d} \alpha \ll_{\ell} \xi N^{1 / \ell} L+ \begin{cases}L^{2} & \text { if } \ell=2 \\
1 & \text { if } \ell>2 .\end{cases}\right.
$$

Proof. The first part was proved in Lemma 2 of [4]. For the second part we argue analogously. We use Corollary 2 of Montgomery-Vaughan [12] with $T=\xi, a_{r}=\log (r) \exp \left(-r^{\ell} / N\right)$ if $r$ is prime, $a_{r}=0$ otherwise and $\lambda_{r}=2 \pi r^{\ell}$. By the Prime Number Theorem we get

$$
\int_{0}^{\xi}\left|\widetilde{V}_{\ell}(\alpha)\right|^{2} \mathrm{~d} \alpha=\sum_{p} \log ^{2}(p) e^{-2 p^{\ell} / N}\left(\xi+\sigma\left(\delta_{p}^{-1}\right)\right) \ll_{\ell} \xi N^{1 / \ell} L+\sum_{p} \log ^{2}(p) p^{1-\ell} e^{-2 p^{\ell} / N}
$$

since $\delta_{r}=\lambda_{r}-\lambda_{r-1} \gg_{\ell} r^{\ell-1}$. The last term is $\ll_{\ell} 1$ if $\ell>2$ and $\ll L^{2}$ otherwise. The second part of Lemma 5 follows.

In the following we will also need a fourth-power average of $\widetilde{S}_{2}(\alpha)$. 
Lemma 6 (Lemma 6 of [5]). We have

$$
\int_{-1 / 2}^{1 / 2}\left|\widetilde{S}_{2}(\alpha)\right|^{4} \mathrm{~d} \alpha \ll N L^{2}
$$

\section{Proof of Theorem 1}

Let $\varepsilon>0$ and $H>2 B$, where

$$
B=B(N, d)=\exp \left(d\left(\frac{\log N}{\log \log N}\right)^{1 / 3}\right),
$$

where $d=d(\varepsilon)>0$ will be chosen later. Recalling (1), we may write

$$
\sum_{n=N+1}^{N+H} e^{-n / N} r_{k}(n)=\int_{-1 / 2}^{1 / 2} \widetilde{V}_{k}(\alpha) \widetilde{V}_{2}(\alpha)^{2} U(-\alpha, H) e(-N \alpha) \mathrm{d} \alpha .
$$

We find it also convenient to set

$$
\widetilde{E}_{\ell}(\alpha):=\widetilde{S}_{\ell}(\alpha)-\frac{\Gamma(1 / \ell)}{\ell z^{1 / \ell}}
$$

Letting $I(B, H):=[-1 / 2,-B / H] \cup[B / H, 1 / 2]$, using Lemma2, recalling that $\Gamma(1 / 2)=\pi^{1 / 2}$, we can write

$$
\begin{aligned}
\sum_{n=N+1}^{N+H} e^{-n / N} r_{k}(n)=\int_{-B / H}^{B / H} & \frac{\pi \Gamma(1 / k)}{4 k z^{1+1 / k}} U(-\alpha, H) e(-N \alpha) \mathrm{d} \alpha \\
& +\int_{-B / H}^{B / H} \frac{\Gamma(1 / k)}{k z^{1 / k}}\left(\widetilde{S}_{2}(\alpha)^{2}-\frac{\pi}{4 z}\right) U(-\alpha, H) e(-N \alpha) \mathrm{d} \alpha \\
& +\int_{-B / H}^{B / H} \widetilde{E}_{k}(\alpha) \widetilde{S}_{2}(\alpha)^{2} U(-\alpha, H) e(-N \alpha) \mathrm{d} \alpha \\
& +\int_{-1 / 2}^{1 / 2} \widetilde{V}_{k}(\alpha)\left(\widetilde{V}_{2}(\alpha)^{2}-\widetilde{S}_{2}(\alpha)^{2}\right) U(-\alpha, H) e(-N \alpha) \mathrm{d} \alpha \\
& +\int_{-1 / 2}^{1 / 2} \widetilde{S}_{2}(\alpha)^{2}\left(\widetilde{V}_{k}(\alpha)-\widetilde{S}_{k}(\alpha)\right) U(-\alpha, H) e(-N \alpha) \mathrm{d} \alpha \\
& +\int_{I(B, H)} \widetilde{S}_{k}(\alpha) \widetilde{S}_{2}(\alpha)^{2} U(-\alpha, H) e(-N \alpha) \mathrm{d} \alpha \\
=\mathscr{I}_{1}+ & +\mathscr{J}_{2}+\mathscr{J}_{3}+\mathscr{J}_{4}+\mathscr{J}_{5}+\mathscr{F}_{6},
\end{aligned}
$$

say. Now we evaluate these terms.

3.1. Evaluation of $\mathscr{F}_{1}$. Using Lemma 3 and (3) we immediately get

$$
\begin{aligned}
\mathscr{g}_{1} & =\frac{\pi \Gamma(1 / k)}{4 k \Gamma(1+1 / k)} \sum_{n=N+1}^{N+H} n^{1 / k} e^{-n / N}+\circlearrowleft_{k}\left(\frac{H}{N}\right)+\circlearrowleft_{k}\left(\int_{B / H}^{1 / 2} \frac{\mathrm{d} \alpha}{\alpha^{2+1 / k}}\right) \\
& =\frac{\pi}{4 e} H N^{1 / k}+\circlearrowleft_{k}\left(H^{2} N^{1 / k-1}+N^{1 / k}+\left(\frac{H}{B}\right)^{1+1 / k}\right) .
\end{aligned}
$$


3.2. Estimation of $\mathscr{F}_{6}$. Using $\widetilde{S}_{k}(\alpha) \ll_{k} N^{1 / k}$, (4), Lemma 5 and a partial integration, we obtain that

$$
\begin{aligned}
\mathscr{F}_{6} & \ll_{k} N^{1 / k} \int_{B / H}^{1 / 2} \frac{\left|\widetilde{S}_{2}(\alpha)\right|^{2}}{\alpha} \mathrm{d} \alpha \\
& \ll_{k} N^{1 / k}\left[\frac{H}{B}\left(\frac{N^{1 / 2} B L}{H}+L^{2}\right)+N^{1 / 2} L+\int_{B / H}^{1 / 2} \frac{N^{1 / 2} \xi L+L^{2}}{\xi^{2}} \mathrm{~d} \xi\right] \\
& \ll_{k} N^{1 / k} L^{2}\left(N^{1 / 2}+\frac{H}{B}\right)
\end{aligned}
$$

which, comparing with (8), is under control for $H=\infty\left(N^{1 / 2} L^{2}\right)$ and $B=\infty\left(L^{2}\right)$ (which is fine thanks to (5)).

3.3. Estimation of $\mathscr{F}_{5}$. By Lemmas 1 and 5, (4) and a partial integration we get

$$
\begin{aligned}
\mathscr{g}_{5} & \ll \int_{-1 / 2}^{1 / 2}\left|\widetilde{S}_{2}(\alpha)\right|^{2}\left|\widetilde{V}_{k}(\alpha)-\widetilde{S}_{k}(\alpha)\right||U(-\alpha, H)| \mathrm{d} \alpha \\
& \ll_{k} H N^{1 /(2 k)} \int_{-1 / H}^{1 / H}\left|\widetilde{S}_{2}(\alpha)\right|^{2} \mathrm{~d} \alpha+N^{1 /(2 k)} \int_{1 / H}^{1 / 2} \frac{\left|\widetilde{S}_{2}(\alpha)\right|^{2}}{\alpha} \mathrm{d} \alpha \\
& \ll_{k} H N^{1 /(2 k)}\left(\frac{N^{1 / 2} L}{H}+L^{2}\right)+N^{1 /(2 k)}\left(N^{1 / 2} L+H L^{2}+\int_{1 / H}^{1 / 2} \frac{N^{1 / 2} \xi L+L^{2}}{\xi^{2}} \mathrm{~d} \xi\right) \\
& \ll_{k} N^{1 /(2 k)}\left(N^{1 / 2}+H\right) L^{2} .
\end{aligned}
$$

which, comparing with $(\underline{8})$, is under control for $H=\infty\left(N^{1 / 2-1 /(2 k)} L^{2}\right)$.

3.4. Estimation of $g_{4}$. Using the identity $f^{2}-g^{2}=2 f(f-g)-(f-g)^{2}$, Lemma 1 and $\widetilde{V}_{k}(\alpha) \ll_{k} N^{1 / k}$, we have

$$
\begin{aligned}
\widetilde{V}_{k}(\alpha)\left(\widetilde{V}_{2}(\alpha)^{2}-\widetilde{S}_{2}(\alpha)^{2}\right) & \ll_{k}\left|\widetilde{V}_{k}(\alpha)\right|\left(\left|\widetilde{V}_{2}(\alpha)\right|\left|\widetilde{V}_{2}(\alpha)-\widetilde{S}_{2}(\alpha)\right|+\left|\widetilde{V}_{2}(\alpha)-\widetilde{S}_{2}(\alpha)\right|^{2}\right) \\
& \ll_{k} N^{1 / 4}\left|\widetilde{V}_{k}(\alpha)\right|\left|\widetilde{V}_{2}(\alpha)\right|+N^{1 / 2+1 / k} .
\end{aligned}
$$

Clearly we have

$$
\begin{aligned}
\mathscr{f}_{4} & \ll_{k} N^{1 / 4} \int_{-1 / 2}^{1 / 2}\left|\widetilde{V}_{k}(\alpha)\right|\left|\widetilde{V}_{2}(\alpha)\right||U(-\alpha, H)| \mathrm{d} \alpha+N^{1 / 2+1 / k} \int_{-1 / 2}^{1 / 2}|U(-\alpha, H)| \mathrm{d} \alpha \\
& =K_{1}+K_{2},
\end{aligned}
$$

say. Using (4) we get

$$
\int_{-1 / 2}^{1 / 2}|U(-\alpha, H)| \mathrm{d} \alpha \ll \int_{-1 / H}^{1 / H} H \mathrm{~d} \alpha+\int_{1 / H}^{1 / 2} \frac{\mathrm{d} \alpha}{\alpha} \ll L
$$

and hence, by (11)-(12), we can write

$$
K_{2} \ll_{k} N^{1 / 2+1 / k} L,
$$

for every $k \geq 1$.

Now we estimate $K_{1}$; depending on $k$, we need to perform different computations. 
Let $k=1$. Using the Cauchy-Schwarz inequality, the Prime Number Theorem, (4) and Lemma 5, we obtain that

$$
\begin{aligned}
K_{1} & \ll N^{1 / 4}\left(\int_{-1 / 2}^{1 / 2}\left|\widetilde{V}_{1}(\alpha)\right|^{2} \mathrm{~d} \alpha\right)^{1 / 2}\left(\int_{-1 / 2}^{1 / 2}\left|\widetilde{V}_{2}(\alpha)\right|^{2}|U(-\alpha, H)|^{2} \mathrm{~d} \alpha\right)^{1 / 2} \\
& \ll N^{3 / 4} L^{1 / 2}\left[H^{2}\left(\frac{N^{1 / 2} L}{H}+L^{2}\right)+N^{1 / 2} L+\int_{1 / H}^{1 / 2} \frac{N^{1 / 2} \xi L+L^{2}}{\xi^{3}} \mathrm{~d} \xi\right]^{1 / 2} \\
& \ll H N^{3 / 4} L^{3 / 2}+H^{1 / 2} N L .
\end{aligned}
$$

Hence, by (11) and (13)-(14), for $k=1$ we get

$$
\mathscr{f}_{4} \ll N^{3 / 2} L+H N^{3 / 4} L^{3 / 2} .
$$

Let $k=2$. Using (4) and Lemma 5 , we obtain that

$$
\begin{aligned}
K_{1} & \ll N^{1 / 4} \int_{-1 / 2}^{1 / 2}\left|\widetilde{V}_{2}(\alpha)\right|^{2}|U(-\alpha, H)| \mathrm{d} \alpha \\
& \ll N^{1 / 4}\left[H\left(\frac{N^{1 / 2} L}{H}+L^{2}\right)+N^{1 / 2} L+\int_{1 / H}^{1 / 2} \frac{N^{1 / 2} \xi L+L^{2}}{\xi^{2}} \mathrm{~d} \xi\right] \\
& \ll H N^{1 / 4} L^{2}+N^{3 / 4} L^{2} .
\end{aligned}
$$

Hence, by (11), (13) and (16), for $k=2$ we get

$$
\mathscr{f}_{4} \ll N L+H N^{1 / 4} L^{2} .
$$

Let $k=3$. Using the Cauchy-Schwarz estimate, (4) and Lemma 5, we obtain that

$$
\begin{aligned}
K_{1} \ll & N^{1 / 4}\left(\int_{-1 / 2}^{1 / 2}\left|\widetilde{V}_{3}(\alpha)\right|^{2}|U(-\alpha, H)| \mathrm{d} \alpha\right)^{1 / 2}\left(\int_{-1 / 2}^{1 / 2}\left|\widetilde{V}_{2}(\alpha)\right|^{2}|U(-\alpha, H)| \mathrm{d} \alpha\right)^{1 / 2} \\
\ll & N^{1 / 4}\left[H\left(\frac{N^{1 / 3} L}{H}+1\right)+N^{1 / 3} L+\int_{1 / H}^{1 / 2} \frac{N^{1 / 3} \xi L+1}{\xi^{2}} \mathrm{~d} \xi\right]^{1 / 2} \\
& \times\left[H\left(\frac{N^{1 / 2} L}{H}+L^{2}\right)+N^{1 / 2} L+\int_{1 / H}^{1 / 2} \frac{N^{1 / 2} \xi L+L^{2}}{\xi^{2}} \mathrm{~d} \xi\right]^{1 / 2} \\
\ll & H N^{1 / 4} L+H^{1 / 2} N^{1 / 2} L+N^{2 / 3} L^{2} .
\end{aligned}
$$

Hence, by (11), (13) and (18), for $k=3$ we get

$$
\mathscr{f}_{4} \ll N^{5 / 6} L+H N^{1 / 4} L+H^{1 / 2} N^{1 / 2} L .
$$

Let $k \geq 4$. By (11), $\widetilde{V}_{k}(\alpha) \ll_{k} N^{1 / k}$ and (12) we can write that

$$
\mathscr{f}_{4} \ll_{k} N^{3 / 4+1 / k} \int_{-1 / 2}^{1 / 2}|U(-\alpha, H)| \mathrm{d} \alpha \ll_{k} N^{3 / 4+1 / k} L .
$$

Summing up, from $(\overline{15}),(17)$ and $(\overline{19})-(\sqrt{20})$ we can write that

$$
\mathscr{J}_{4} \ll_{k} E(k)
$$

where $E(k)$ is defined in (2). 
3.5. Estimation of $\mathscr{F}_{2}$. Now we estimate $\mathscr{F}_{2}$. Using the identity $f^{2}-g^{2}=2 f(f-g)-(f-g)^{2}$ we obtain

$$
\mathscr{g}_{2} \ll_{k} \int_{-B / H}^{B / H}\left|\widetilde{E}_{2}(\alpha)\right| \frac{|U(\alpha, H)|}{|z|^{1 / 2+1 / k}} \mathrm{~d} \alpha+\int_{-B / H}^{B / H}\left|\widetilde{E}_{2}(\alpha)\right|^{2} \frac{|U(\alpha, H)|}{|z|^{1 / k}} \mathrm{~d} \alpha=I_{1}+I_{2},
$$

say. Using (3), (4), Lemma 4 and a partial integration argument we obtain that, for every $\varepsilon>0$, there exists $c_{1}=c_{1}(\varepsilon)>0$ such that

$$
I_{2} \ll_{k} H N^{1 / k} \int_{-B / H}^{B / H}\left|\widetilde{E}_{2}(\alpha)\right|^{2} \mathrm{~d} \alpha \ll_{k} H N^{1 / k} \exp \left(-c_{1}\left(\frac{L}{\log L}\right)^{1 / 3}\right)
$$

provided that $H \geq B N^{7 / 12+\varepsilon}$. Using the Cauchy-Schwarz inequality and arguing as for $I_{2}$ we get

$$
I_{1} \ll_{k} H\left(\int_{-B / H}^{B / H}\left|\widetilde{E}_{2}(\alpha)\right|^{2} \mathrm{~d} \alpha\right)^{1 / 2}\left(\int_{-B / H}^{B / H} \frac{\mathrm{d} \alpha}{\alpha^{1+2 / k}}\right)^{1 / 2} \ll_{k} H N^{1 / k} \exp \left(-\frac{c_{1}}{2}\left(\frac{L}{\log L}\right)^{1 / 3}\right),
$$

provided that $H \geq B N^{7 / 12+\varepsilon}$. Inserting (23)-(24) into (22) we finally obtain

$$
\mathscr{I}_{2} \ll_{k} H N^{1 / k} \exp \left(-\frac{c_{1}}{2}\left(\frac{L}{\log L}\right)^{1 / 3}\right),
$$

provided that $H \geq B N^{7 / 12+\varepsilon}$.

3.6. Estimation of $\mathscr{F}_{3}$. Now we estimate $\mathscr{F}_{3}$. By the Cauchy-Schwarz inequality, (4), Lemmas 6 and Lemma 4, we obtain that, for every $\varepsilon>0$, there exists $c_{1}=c_{1}(\varepsilon)>0$ such that

$$
\begin{aligned}
\mathscr{J}_{3} & \ll_{k}\left(\int_{-1 / 2}^{1 / 2}\left|\widetilde{S}_{2}(\alpha)\right|^{4} \mathrm{~d} \alpha\right)^{1 / 2}\left(\int_{-B / H}^{B / H}\left|\widetilde{E}_{k}(\alpha)\right|^{2}|U(\alpha, H)|^{2} \mathrm{~d} \alpha\right)^{1 / 2} \\
& \ll_{k} H N^{1 / 2} L\left(\int_{-B / H}^{B / H}\left|\widetilde{E}_{k}(\alpha)\right|^{2} \mathrm{~d} \alpha\right)^{1 / 2} \ll_{k} H N^{1 / k} \exp \left(-\frac{c_{1}}{2}\left(\frac{L}{\log L}\right)^{1 / 3}\right),
\end{aligned}
$$

provided that $H \geq B N^{1-5 /(6 k)+\varepsilon}$.

3.7. Final words. Let $k \geq 2$. By (17)-(10), (21) and (25)-(26) we have that, for every $\varepsilon>0$, there exists $c_{1}=c_{1}(\varepsilon)>0$ such that

$$
\begin{aligned}
\sum_{n=N+1}^{N+H} e^{-n / N} r_{k}(n) & =\frac{\pi}{4 e} H N^{1 / k} \\
& +\sigma_{k}\left(H N^{1 / k} \exp \left(-\frac{c_{1}}{2}\left(\frac{L}{\log L}\right)^{1 / 3}\right)+\frac{H}{B} N^{1 / k} L^{2}+E(k)\right)
\end{aligned}
$$

provided that $H \geq B N^{1-5 /(6 k)+\varepsilon}$. The second error term is dominated by the first one by choosing $d=c_{1}$ in (5). So from now on we have $H \geq N^{1-5 /(6 k)+\varepsilon}$ for $k \geq 2$. The third error term in (27) is now dominated by the first.

Let $k=1$. In this case (27) holds provided that $H \geq B N^{7 / 12+\varepsilon}$ and the second error term is dominated by the first one by choosing $d=c_{1}$ in (5). Hence, for $k=1$, we get that $H \geq N^{7 / 12+\varepsilon}$. The third error term in (27) is now dominated by the first.

Summing up, for every $k \geq 1$ we can write that, for every $\varepsilon>0$, there exists $C=C(\varepsilon)>0$ such that

$$
\sum_{n=N+1}^{N+H} e^{-n / N} r_{k}(n)=\frac{\pi}{4 e} H N^{1 / k}+\sigma_{k}\left(H N^{1 / k} \exp \left(-C\left(\frac{L}{\log L}\right)^{1 / 3}\right)\right)
$$


provided that $H \geq N^{1-5 /(6 k)+\varepsilon}$ for $k \geq 2$ and $H \geq N^{7 / 12+\varepsilon}$ for $k=1$. From $e^{-n / N}=$ $e^{-1}+\mathcal{O}(H / N)$ for $n \in[N+1, N+H], 1 \leq H \leq N$, we get that, for every $\varepsilon>0$, there exists $C=C(\varepsilon)>0$ such that

$$
\sum_{n=N+1}^{N+H} r_{k}(n)=\frac{\pi}{4} H N^{1 / k}+\circlearrowleft_{k}\left(H N^{1 / k} \exp \left(-C\left(\frac{L}{\log L}\right)^{1 / 3}\right)\right)+\circlearrowleft_{k}\left(\frac{H}{N} \sum_{n=N+1}^{N+H} r_{k}(n)\right)
$$

provided that $H \leq N, H \geq N^{1-5 /(6 k)+\varepsilon}$ for $k \geq 2$ and $H \geq N^{7 / 12+\varepsilon}$ for $k=1$. Using $e^{n / N} \leq e^{2}$ and (27), the last error term is $\ll_{k} H^{2} N^{1 / k-1}$. Hence we get

$$
\sum_{n=N+1}^{N+H} r_{k}(n)=\frac{\pi}{4} H N^{1 / k}+\sigma_{k}\left(H N^{1 / k} \exp \left(-C\left(\frac{L}{\log L}\right)^{1 / 3}\right)\right)
$$

uniformly for $N^{1-5 /(6 k)+\varepsilon} \leq H \leq N^{1-\varepsilon}$ if $k \geq 2$ and for $N^{7 / 12+\varepsilon} \leq H \leq N^{1-\varepsilon}$ if $k=1$. Theorem 1 follows.

\section{Proof of Theorem 2}

Let $k \geq 2, H \geq 2, H=o(N)$ be an integer. We recall that we set $L=\log N$ for brevity. From now on we assume that RH holds. we may write

$$
\sum_{n=N+1}^{N+H} e^{-n / N} r_{k}(n)=\int_{-1 / 2}^{1 / 2} \widetilde{V}_{k}(\alpha) \widetilde{V}_{2}(\alpha)^{2} U(-\alpha, H) e(-N \alpha) \mathrm{d} \alpha .
$$

In this conditional case we can simplify the setting. Using Lemma_, recalling definition (6) and that $\Gamma(1 / 2)=\pi^{1 / 2}$, we can write

$$
\begin{aligned}
\sum_{n=N+1}^{N+H} e^{-n / N} r_{k}(n)=\int_{-1 / 2}^{1 / 2} & \frac{\pi \Gamma(1 / k)}{4 k z^{1+1 / k}} U(-\alpha, H) e(-N \alpha) \mathrm{d} \alpha \\
& +\int_{-1 / 2}^{1 / 2} \frac{\Gamma(1 / k)}{k z^{1 / k}}\left(\widetilde{S}_{2}(\alpha)^{2}-\frac{\pi}{4 z}\right) U(-\alpha, H) e(-N \alpha) \mathrm{d} \alpha \\
& +\int_{-1 / 2}^{1 / 2} \widetilde{E}_{k}(\alpha) \widetilde{S}_{2}(\alpha)^{2} U(-\alpha, H) e(-N \alpha) \mathrm{d} \alpha \\
& +\int_{-1 / 2}^{1 / 2} \widetilde{V}_{k}(\alpha)\left(\widetilde{V}_{2}(\alpha)^{2}-\widetilde{S}_{2}(\alpha)^{2}\right) U(-\alpha, H) e(-N \alpha) \mathrm{d} \alpha \\
& +\int_{-1 / 2}^{1 / 2} \widetilde{S}_{2}(\alpha)^{2}\left(\widetilde{V}_{k}(\alpha)-\widetilde{S}_{k}(\alpha)\right) U(-\alpha, H) e(-N \alpha) \mathrm{d} \alpha \\
=\mathscr{I}_{1} & +\mathscr{I}_{2}+\mathscr{I}_{3}+\mathscr{I}_{4}+\mathscr{I}_{5}
\end{aligned}
$$

say. Now we evaluate these terms.

4.1. Evaluation of $\mathscr{I}_{1}$. Using Lemma 3 we immediately get

$$
\mathscr{I}_{1}=\frac{\pi \Gamma(1 / k)}{4 k \Gamma(1+1 / k)} \sum_{n=N+1}^{N+H} n^{1 / k} e^{-n / N}+\circlearrowleft_{k}\left(\frac{H}{N}\right)=\frac{\pi}{4 e} H N^{1 / k}+\circlearrowleft_{k}\left(H^{2} N^{1 / k-1}+N^{1 / k}\right) .
$$


4.2. Estimation of $\mathscr{I}_{5}$. Clearly $\mathscr{I}_{5}=\mathscr{F}_{5}$ of section 3.3. Hence we have that

$$
\mathscr{I}_{5} \ll_{k} N^{1 /(2 k)}\left(N^{1 / 2}+H\right) L^{2}
$$

which, comparing with (29), is under control for $H=\infty\left(N^{1 / 2-1 /(2 k)} L^{2}\right)$.

4.3. Estimation of $\mathscr{I}_{4}$. Clearly $\mathscr{I}_{4}=\mathscr{F}_{4}$ of section 3.4. Hence we have that

$$
\mathscr{I}_{4} \ll_{k} E(k)
$$

where $E(k)$ is defined in (2).

4.4. Estimation of $\mathscr{I}_{2}$. Now we estimate $\mathscr{I}_{2}$. Using the identity $f^{2}-g^{2}=2 f(f-g)-(f-g)^{2}$ we obtain

$$
\mathscr{I}_{2} \ll_{k} \int_{-1 / 2}^{1 / 2}\left|\widetilde{E}_{2}(\alpha)\right| \frac{|U(\alpha, H)|}{|z|^{1 / 2+1 / k}} \mathrm{~d} \alpha+\int_{-1 / 2}^{1 / 2}\left|\widetilde{E}_{2}(\alpha)\right|^{\mid} \frac{|U(\alpha, H)|}{|z|^{1 / k}} \mathrm{~d} \alpha=J_{1}+J_{2},
$$

say. Using (3), (4), Lemma 4 and a partial integration argument we obtain

$$
\begin{aligned}
J_{2} & \ll_{k} H N^{1 / k} \int_{-1 / N}^{1 / N}\left|\widetilde{E}_{2}(\alpha)\right|^{2} \mathrm{~d} \alpha+H \int_{1 / N}^{1 / H}\left|\widetilde{E}_{2}(\alpha)\right|^{2} \frac{\mathrm{d} \alpha}{\alpha^{1 / k}}+\int_{1 / H}^{1 / 2}\left|\widetilde{E}_{2}(\alpha)\right|^{2} \frac{\mathrm{d} \alpha}{\alpha^{1+1 / k}} \\
& \ll_{k} H N^{1 / k-1 / 2} L^{2}+H^{1 / k} N^{1 / 2} L^{2} \ll_{k} H^{1 / k} N^{1 / 2} L^{2} .
\end{aligned}
$$

For $J_{1}$ we need few cases. Let $k \geq 3$. Using the Cauchy-Schwarz inequality and arguing as for $J_{2}$ we get

$$
\begin{aligned}
J_{1} & \ll_{k} H N^{1 / 2+1 / k}\left(\int_{-1 / N}^{1 / N} \mathrm{~d} \alpha\right)^{1 / 2}\left(\int_{-1 / N}^{1 / N}\left|\widetilde{E}_{2}(\alpha)\right|^{2} \mathrm{~d} \alpha\right)^{1 / 2} \\
& +H\left(\int_{1 / N}^{1 / H} \frac{\mathrm{d} \alpha}{\alpha^{2 / k}}\right)^{1 / 2}\left(\int_{1 / N}^{1 / H}\left|\widetilde{E}_{2}(\alpha)\right|^{2} \frac{\mathrm{d} \alpha}{\alpha}\right)^{1 / 2}+\left(\int_{1 / H}^{1 / 2} \frac{\mathrm{d} \alpha}{\alpha^{2+2 / k}}\right)^{1 / 2}\left(\int_{1 / H}^{1 / 2}\left|\widetilde{E}_{2}(\alpha)\right|^{2} \frac{\mathrm{d} \alpha}{\alpha}\right)^{1 / 2} \\
& \ll_{k} H N^{1 / k-1 / 4} L+H^{1 / 2+1 / k} N^{1 / 4} L\left(1+\int_{1 / N}^{1 / H} \frac{\mathrm{d} \xi}{\xi}\right)^{1 / 2}+H^{1 / 2+1 / k} N^{1 / 4} L\left(1+\int_{1 / H}^{1 / 2} \frac{\mathrm{d} \xi}{\xi}\right)^{1 / 2} \\
& \ll_{k} H N^{1 / k-1 / 4} L+H^{1 / 2+1 / k} N^{1 / 4} L^{3 / 2} \ll H^{1 / 2+1 / k} N^{1 / 4} L^{3 / 2} .
\end{aligned}
$$

For $k=2$ arguing as before we get

$$
J_{1} \ll H N^{1 / 4} L^{2} .
$$

Combining (32)-(35), and assuming $H \geq N^{1 / 2}$, we finally obtain

$$
\mathscr{I}_{2} \ll_{k} H^{1 / 2+1 / k} N^{1 / 4} L^{2}
$$

for every $k \geq 2$.

4.5. Estimation of $\mathscr{I}_{3}$. Now we estimate $\mathscr{I}_{3}$. By the Cauchy-Schwarz inequality, (4) and Lemma 6 we obtain

$$
\begin{aligned}
\mathscr{I}_{3} & \ll_{k}\left(\int_{-1 / 2}^{1 / 2}\left|\widetilde{S}_{2}(\alpha)\right|^{4} \mathrm{~d} \alpha\right)^{1 / 2}\left(\int_{-1 / 2}^{1 / 2}\left|\widetilde{E}_{k}(\alpha)\right|^{2}|U(\alpha, H)|^{2} \mathrm{~d} \alpha\right)^{1 / 2} \\
& \ll_{k} N^{1 / 2} L\left(H^{2} \int_{-1 / H}^{1 / H}\left|\widetilde{E}_{k}(\alpha)\right|^{2} \mathrm{~d} \alpha+\int_{1 / H}^{1 / 2}\left|\widetilde{E}_{k}(\alpha)\right|^{2} \frac{\mathrm{d} \alpha}{\alpha^{2}}\right)^{1 / 2} \ll_{k} H^{1 / 2} N^{1 / 2+1 /(2 k)} L^{2},
\end{aligned}
$$

where in the last step we used Lemma 4 and a partial integration argument. 
4.6. Final words. By (28)-( $\sqrt{31})$ and $(\sqrt{36})-(\sqrt{37})$, we can finally write for $H \geq N^{1 / 2}$ that

$$
\sum_{n=N+1}^{N+H} e^{-n / N} r_{k}(n)=\frac{\pi}{4 e} H N^{1 / k}+\sigma_{k}\left(H^{2} N^{1 / k-1}+H^{1 / 2} N^{1 / 2+1 /(2 k)} L^{2}+E(k)\right)
$$

which is an asymptotic formula for $\infty\left(N^{1-1 / k} L^{4}\right) \leq H \leq o(N)$. From $e^{-n / N}=e^{-1}+\mathcal{O}(H / N)$ for $n \in[N+1, N+H], 1 \leq H \leq N$, we get

$$
\begin{aligned}
\sum_{n=N+1}^{N+H} r_{k}(n) & =\frac{\pi}{4 e} H N^{1 / k}+\sigma_{k}\left(H^{2} N^{1 / k-1}+H^{1 / 2} N^{1 / 2+1 /(2 k)} L^{2}+E(k)\right) \\
& +\sigma_{k}\left(\frac{H}{N} \sum_{n=N+1}^{N+H} r_{k}(n)\right) .
\end{aligned}
$$

Using $e^{n / N} \leq e^{2}$ and $(38)$, the last error term is $\ll_{k} H^{2} N^{1 / k-1}+H^{3 / 2} N^{-1 / 2+1 /(2 k)} L^{2}+H N^{-1} E(k)$. Hence we get

$$
\sum_{n=N+1}^{N+H} r_{k}(n)=\frac{\pi}{4 e} H N^{1 / k}+\sigma_{k}\left(H^{2} N^{1 / k-1}+H^{1 / 2} N^{1 / 2+1 /(2 k)} L^{2}+E(k)\right),
$$

uniformly for $\infty\left(N^{1-1 / k} L^{4}\right) \leq H \leq o(N)$. Theorem 2 follows.

\section{REFERENCES}

[1] J. Brüdern, A ternary problem in additive prime number theory, in: From Arithmetic to Zeta-Functions. Number Theory in Memory of Wolfgang Schwarz (J. Sanders et al, ed.), Springer, 2016, pp. 57-81.

[2] G. Harman and A.V. Kumchev, On sums of squares of primes II, J. Number Theory 130 (2010), $1969-2002$.

[3] L. K. Hua, Some results in the additive prime number theory, Quart. J. Math. Oxford 9 (1938), 68-80.

[4] A. Languasco and A. Zaccagnini, Short intervals asymptotic formulae for binary problems with primes and powers, II: density 1, Monatsh. Math. 181 (2016), 419-435.

[5] A. Languasco and A. Zaccagnini, Sum of one prime and two squares of primes in short intervals, J. Number Theory 159 (2016), 45-58.

[6] A. Languasco and A. Zaccagnini, Short intervals asymptotic formulae for binary problems with primes and powers, I: density 3/2, Ramanujan J. 42 (2017), no. 2, 371-383.

[7] A. Languasco and A. Zaccagnini, Sums of four prime cubes in short intervals, Submitted for publication. Arxiv preprint 1705.04457, 2017.

[8] M.C. Leung and M.C. Liu, On generalized quadratic equations in three prime variables, Monatsh. Math. 115 (1993), 133-167.

[9] T. Li, On sums of squares of primes and a kth power of prime, Rocky Mountain J. Math. 42 (2012), $201-222$.

[10] G.-S. Lü, Note on a result of Hua, Adv. Math. (China) 35 (2006), 343-349.

[11] H. L. Montgomery, Ten Lectures on the Interface Between Analytic Number Theory and Harmonic Analysis, CBMS Regional Conference Series in Mathematics, vol. 84, A.M.S., 1994.

[12] H. L. Montgomery and R. C. Vaughan, Hilbert's inequality, J. London Math. Soc. 8 (1974), $73-82$.

[13] W. Schwarz, Zur Darstellung von Zahlen durch Summen von Primzahlpotenzen. II., J. Reine Angew. Math. $206(1961), 78-112$.

[14] R. C. Vaughan, The Hardy-Littlewood method, second ed., Cambridge U. P., 1997.

[15] L. Zhao, The additive problem with one prime and two squares of primes, J. Number Theory 135 (2014), 8-27. 
Alessandro Languasco

Università di Padova

Dipartimento di Matematica

"Tullio Levi-Civita"

Via Trieste 63

35121 Padova, Italy

e-mail: alessandro.languasco@unipd.it
Alessandro Zaccagnini

Università di Parma

Dipartimento di Scienze Matematiche,

Fisiche e Informatiche

Parco Area delle Scienze, 53/a

43124 Parma, Italy

e-mail: alessandro.zaccagnini@unipr.it 